\title{
Individual versus group housing affects nociception independently of housing status during development
}

\author{
LESLIE M. SCHWANDT \\ University of Minnesota, Minneapolis, Minnesota
}

\section{(J. Bruce Overmier, Sponsor)}

\begin{abstract}
Individually housed rats displayed longer nociceptive latencies than did group-housed animals both before and after very brief, mild electric shocks. The lack of effect of mild shock in changing latencies indicates that individual housing did not result in a stress-induced hypoalgesia reinstateable by a mild stressor, itself insufficient to cause hypoalgesia in normal animals. The longer latencies were entirely a function of the housing condition current at the time of testing. No carryover effects occurred from the housing condition that was in effect immediately after weaning. Therefore, it is likely that no critical period exists for nociceptive sensitivity. The longer latencies support the hypothesis that individual housing is stressful in rats. These results may be of interest to those planning experiments or comparing results across laboratories on stress-induced hypoalgesia.
\end{abstract}

Individually housed animals are reported to have multiple deficits on associative, behavioral, and physiological measures. Among these are learning deficits in shuttle avoidance (Melzack \& Scott, 1957), in spatial and nonspatial escape in the Morris water maze (Wade \& Maier, 1986), in foraging in a radial arm maze (Einon, 1980), in appetitive learning in a Hebb-Williams maze (Dalyrymple-Alford \& Benton, 1984), and in acquiring the win-stay, lose-shift strategy in two-lever alternation schedules for food reinforcement (Morgan, Einon, \& Nicholas, 1975). Individually housed animals also show increases in aggressiveness (Brain \& Benton, 1983) and slower emergence into a novel environment (DalyrympleAlford \& Benton, 1984). Some of the behavioral deficits require early isolation and are not reversed by later group housing (e.g., deficits in the win-stay, lose-shift strategy; radial arm maze; and Hebb-Williams maze learning), and other deficits (e.g., increased aggression and slower emergence into a novel environment) occur regardless of when the individual housing has taken place and can be reversed by later group housing. Individually housed subjects display decreased sensitivity to morphine (Pugliosi-Allegra $\&$ Oliverio, 1983), lowered nociceptive responsiveness

This research was conducted in partial fulfillment of the requirements of the PhD degree in the Department of Psychology, University of Minnesota, Minneapolis. It was supported by a National Science Foundation graduate fellowship and by the Center for Research in Learning, Perception, and Cognition. I would like to thank J. Bruce Overmier, Kory Schuh, and Lisa Savage for many helpful discussions, and K. Schuh, L. Savage, and Juliann Furdek for aid in conducting this study. Correspondence should be addressed to L. M. Schwandt, Clinical Pharmacology Research Branch, NIH/NIDA/Addiction Research Center, P.O. Box 5180, Baltimore, MD 21224. to pin-prick and heat stimuli (Melzack \& Scott, 1957), decreases in shock-elicited jumping (Nishikawa \& Tanaka, 1978), and increases in baseline pain thresholds on the tail-flick test (Gentsch, Lichtsteiner, Fritschknecht, Feer, \& Siegfried, 1988; Pugliosi-Allegra \& Oliverio, 1983). Pugliosi-Allegra and Oliverio reported that the longer baseline latencies of individually housed rats may decrease the effectiveness of immobilization for inducing hypoalgesia. These findings have been attributed to abnormalities in stress responsiveness for the individually housed animals, but this may be an oversimplification, because the effects of individual housing on adrenocortical activity are equivocal (see Brain \& Benton, 1979, for review). Nevertheless, research does clearly indicate that the housing conditions of animals before an experiment may affect the outcome.

The present experiment was designed to confirm the effect of housing on baseline spinal hypoalgesia and to assess its reversibility by changes in housing. That is, it determines whether effects of individual housing are temporary or permanent if they are induced at an early age. Also of interest was whether differential housing modulated stress-induced hypoalgesia.

Animals received housing in either group or individual cages during development and were then rehoused at adulthood into either the same or the opposite condition. This procedure provided four groups for comparison: those individually housed during both development and adulthood (I-I), those individually housed during development and group housed during adulthood (I-G), those group housed during development and individually housed in adulthood (G-I), and those group housed during development and adulthood (G-G). With this method, one can disentangle the effects on hypoalgesia of early rear- 
ing conditions from those of adult housing conditions at the time of testing.

Testing for nociceptive sensitivity included baseline trials, in which latency to flick the tail away from a thermal stimulus was measured without any drug or stressor manipulation, and trials following three brief $(0.75-\mathrm{sec})$ 1 -mA shocks with a 20 -sec intershock interval. These shocks were used because they were insufficient to generate hypoalgesia by themselves but had been reported as reinstating the hypoalgesia of subjects previously sensitized by some challenge (Jackson, Maier, \& Coon, 1979). If differential housing led to stress-induced hypoalgesia in some of the subjects, these shocks might be expected to reinstate that hypoalgesia. Although it was hypothesized that animals individually housed postweaning would have longer latencies than would animals group housed postweaning, it was not clear whether this change might depend on housing during a critical period or whether individual housing at any time would result in the baseline increase, nor was it clear whether reinstating shocks would augment the difference between individually housed and group-housed animals during rearing.

\section{METHOD}

\section{Subjects}

The subjects were 28 male Holtzman rats. They were maintained on an ad-lib schedule of food and water and $24 \mathrm{~h}$ light. They reached the laboratory immediately after weaning at 21 days of age, when they were randomly divided into two equal-sized groups and assigned to individual or group cages. The group condition consisted of 3 animals per cage in four cages and 2 animals in one cage. The subjects were maintained undisturbed in these conditions for 15 weeks before testing occurred, with one exception. Twelve weeks into the study, 1 grouped subject was failing to gain weight and, despite supplementary feeding for $2 \mathrm{~h}$ per day in a private cage, was lost from the experiment at the time of rehousing after the first testing. At the time of first testing, this animal weighed $299 \mathrm{~g}$, whereas the rest of the animals ranged from 514 to $767 \mathrm{~g}$. Following testing of tail nociceptive sensitivity, the subjects were rehoused randomly (with the constraint of approximately equal-sized groups) into either individual or group cages for 1 month: the G-I treatment condition had 6 rats, and the G-G, G-I, and I-I conditions had 7 rats each. At the second testing, the subjects weighed between 559 and $779 \mathrm{~g}$.

\section{Apparatus}

The individual cages measured $18 \times 25 \times 18.5 \mathrm{~cm}$, were of wire mesh, and contained one food hopper and water bottle on the front. The group cages were of the same material and measured $54 \times 25 \times 18.5 \mathrm{~cm}$ for the cages with 3 rats and $36 \times 25 \times 18.5 \mathrm{~cm}$ for the cage with 2 rats. The amount of cage space per animal was equal in both conditions. The group cages had two food hoppers and water bottles on the front of each cage.

Two opaque Plexiglas tubes were used to restrain the animals lightly during testing. The tubes were $22 \mathrm{~cm}$ long and had an internal diameter of $6.8 \mathrm{~cm}$. They were raised $6.7 \mathrm{~cm}$ off the ground and contained 22 ventilation holes $(0.8 \mathrm{~cm}$ in diameter). The fronts of the tubes were sealed by a sheet of opaque Plexiglas. The back was closed by a sliding Plexiglas gate with a slit $2.8 \mathrm{~cm}$ wide and $1.3 \mathrm{~cm}$ from the bottom of the tube through which the subject's tail protruded.

Analgesic testing was conducted with the use of two identical radiant heat tail-flick devices. A 375-W movie bulb (Sylvania, Type EBR) was positioned above the tail. A condenser lens below the bulb focused the light onto the subject's tail approximately $15 \mathrm{~cm}$ from its tip. The rat's tail rested on an aluminum block with a V-shaped groove $7.2 \mathrm{~cm}$ long, $1 \mathrm{~cm}$ wide, and $0.5 \mathrm{~cm}$ deep cut into it. A photocell below the groove automatically terminated a trial when the subject flicked its tail out of the groove at least $0.5 \mathrm{~cm}$. The trial length was automatically timed to the nearest $0.01 \mathrm{sec}$.

In addition, shocks were administered through fixed tail electrodes formed from two modified fuse clips, $1 \mathrm{~cm}$ apart. The electrodes were coated with electrode paste and attached to the subject's tail with adhesive tape. A 600-V transformer in series with an autotransformer and a $100-\mathrm{K} \Omega$ resistor was used to generate the three $0.75-\mathrm{sec}, 1.0-\mathrm{mA}$ shocks with an intertrial interval of $20 \mathrm{sec}$. Testing was conducted in an isolated room with a background white noise level of about $62 \mathrm{dBA}$. Room temperature was approximately $22^{\circ} \pm .8^{\circ} \mathrm{C}$.

\section{Procedure}

Phase 1 consisted of the initial group or individual housing assignment and lasted 15 weeks. Phase 2 consisted of hypoalgesia testing, in which subjects were placed in restraining tubes and allowed to acclimate for $15 \mathrm{~min}$. Four baseline tail-flick trials were administered at 2 min intervals. Following the last baseline trial, the electrodes were taped to the animals' tails and the shocks were administered. The electrodes were removed after the last shock, and tail-flick testing began 2 min later and continued at 2-min intervals until 10 min postshock, as is common in such assessments (e.g., Grau, 1987). A cutoff of $12 \mathrm{sec}$ was used to protect against tissue damage. Because of evidence that circadian rhythmicity affects the body temperature of subjects (Gordon, 1990) and that the tail-flick response occurs when thermal nociceptors reach a critical threshold temperature (Ness \& Gebhart, 1986), the time of day at which subjects are tested might affect the observed nociceptive latency. To control for this, subjects were randomly assigned to testing periods, with the restriction that the average time tested must be the same for each condition. All testing took place between 0830 and $1530 \mathrm{~h}$.

Phase 3 involved rehousing subjects randomly into individual or group housing. This condition lasted for 1 month. This length of rehousing was chosen on the basis of past differential housing studies carried out with 20-30 days rehousing at adulthood, which have yielded effects of differential housing on maze running and open field activity (DalrympleAlford \& Benton, 1984), spatial memory in an Olton radial arm maze (Einon, 1980), sensitivity to pharmacological manipulations (Einon \& Sahakian, 1979), and stress-induced aggression (Goldsmith, Brain, \& Benton, 1978).

In Phase 4, hypoalgesia testing was repeated as in Phase 2, except that the tail-flick latency cutoff was reduced to $8 \mathrm{sec}$, which was substantially longer than the longest group mean value in Phase 2.

\section{RESULTS}

Individually housed animals had reduced pain sensitivity, and this reduction was a function of the immediate housing condition at the time of testing. No carryover effects occurred from the conditions that the animals had received during rearing and development.

The analysis of Phase 2 showed that differential housing altered latencies to the thermal stimulus $[F(1,26)=$ $9.60, p<.05]$, with individually housed rats having longer latencies than group housed rats. Neither trials $[F(5,130)=1.02]$ nor the interaction of housing condition $\times$ trials $[F(5,130)=2.16]$ affected latencies, indicating that the brief, mild shocks did not influence latency to flick the tail from heat; and there was no shock-induced facilitation of hypoalgesia in the individually housed animals. Figure 1 shows mean data for the two housing conditions. For each trial, the individually housed subjects had higher mean latencies than did the grouped animals. ${ }^{1}$ Although there was no overall interaction between housing condition and trials, the reader may notice that $8 \mathrm{~min}$ after shock, the individually housed animals appeared to increase in latency, and the group-housed animals, to de- 

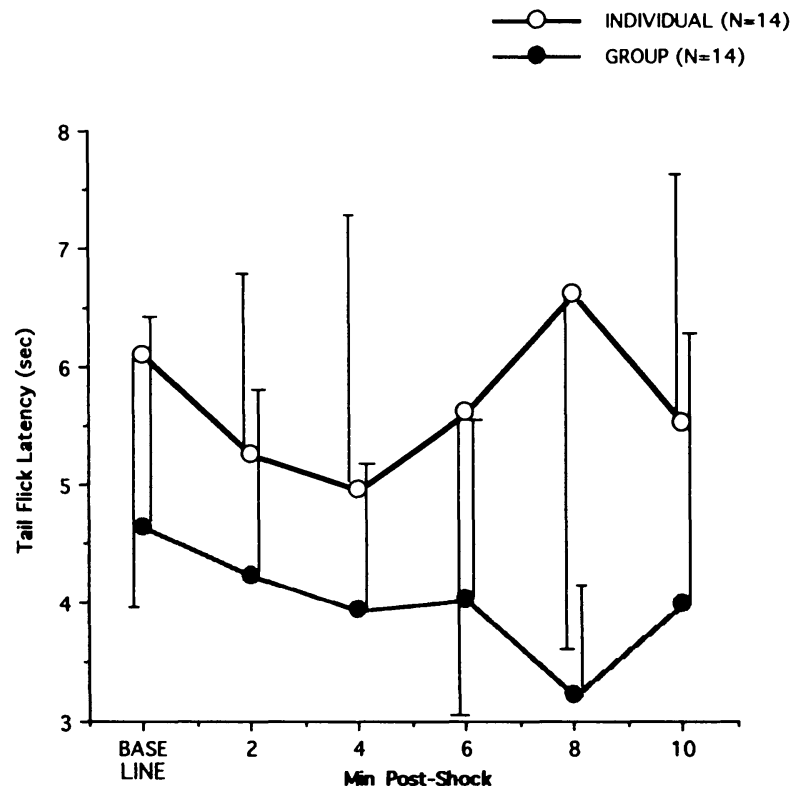

Figure 1. Effect of housing condition immediately postrearing on nociceptive latency. Standard deviations are provided.

crease. A post hoc analysis of changes from baseline using Tukey's HSD indicated that this was not a significant trend $[q(2,26)=2.29]$.

Following the second period of differential housing, the housing condition at the time of testing again determined latency $[F(1,23)=4.48, p<.05]$, with individually housed animals having longer latencies than grouped animals. Prior housing during development had no effect on later latencies $[F(1,23)=0.02]$, and there was no interaction between the two housing conditions $[F(1,23)=.002]$. Again, neither trials nor any interaction with trials reached significance $[F(5,115)=1.40$ for trials; $F(5,115)=0.33$ for prior housing $\times$ trials; $F(5,115)=0.34$ for current housing $\times$ trials; and $F(5,115)=1.00$ for the three-way interaction of conditions and trials]. ${ }^{2}$ The absence of an increase in latency after the brief shocks indicated that they did not reactivate previously elicited hypoalgesic mechanisms, as described by Jackson et al. (1979).

Figure 2 shows the nociceptive latencies of each of the four groups and of groups collapsed across current adult housing condition in the second phase. The subjects currently individually housed have longer mean latencies regardless of how they were housed initially after weaning; and there were no carryover effects of housing during the critical postweaning period.

\section{DISCUSSION}

Our results replicate previous findings of lower pain sensitivity in individually housed animals. Here, we additionally explore animals across two housing phases. The experiment extends the earlier finding by showing that the effect is due to a temporally local housing condition and not some permanent alteration in reactivity induced by isolation during some critical period (viz., Lessac \& Solomon, 1969, vs. Melzack \& Scott, 1957).

The results of this experiment demonstrate that the present procedures and equipment were sensitive to the effects of a psychologically relevant manipulation on changes in pain sensitivity.

Differential housing produced differences in latency, with the individually housed animals having longer latencies than did the grouped animals regardless of the time when differential housing occurred. No evidence for a critical period for the effect existed, and differences in latencies were reversed by shifting animals to the opposite housing condition.

It is consistent with earlier research (e.g., Gentsch et al., 1988; Nishikawa \& Tanaka, 1978; and Pugliosi-Allegra \& Oliverio, 1983) that individually housed subjects had lower pain sensitivity than did

A.

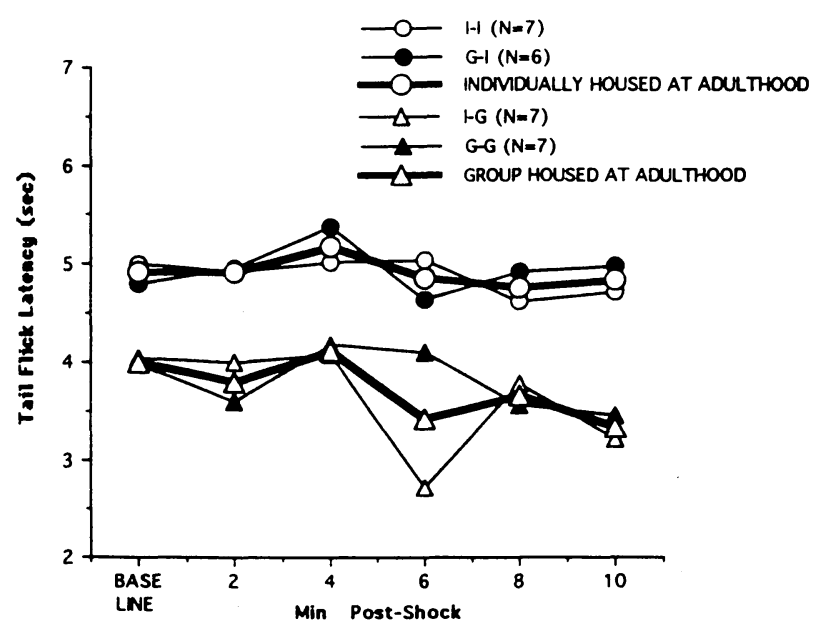

B.
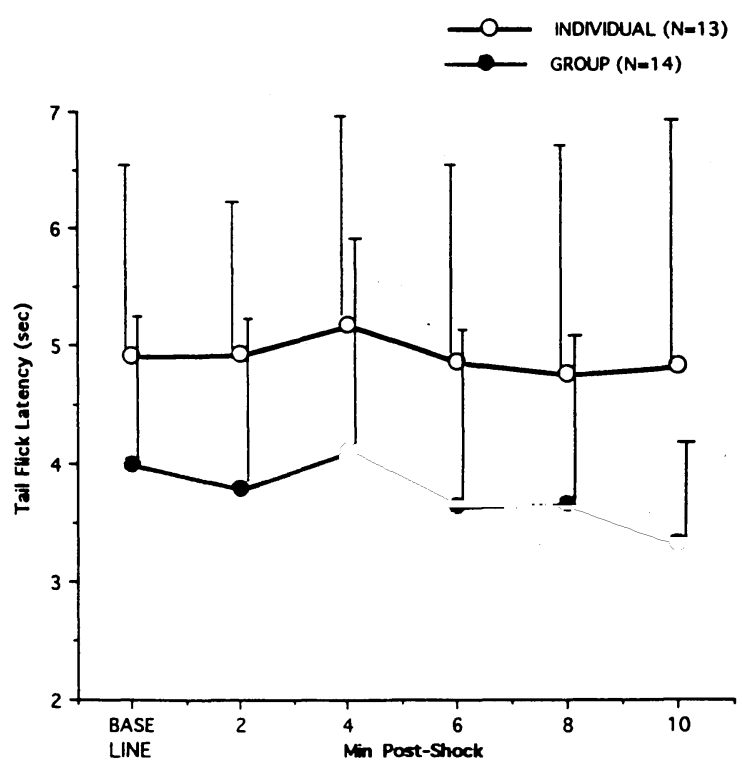

Figure 2. Differential housing effects on nociceptive latency at adulthood after housing reassignments. I, individually housed; G, group housed. Standard deviations are provided. 
grouped subjects. This effect obtained whether individual housing $\propto c-$ curred during development or at adulthood without a critical period. Whether differential housing occurred immediately after weaning or at adulthood, the same effect on nociceptive latency resulted.

Because pain sensitivity is not a stable trait (i.e., it can be modified by environmental events such as pain and stress, Basbaum \& Fields, 1984; Watkins \& Mayer, 1982), this lack of a critical period is probably not surprising. The syndrome of changes resulting from individual housing has been called isolation stress, and the observation that feral rats do live in colonies supports the claim that individual housing may be stressful (Brain \& Benton, 1979), as does the lower pain sensitivity reported here. Data are inconsistent on individual housing and on the hallmark of a stress response, increased adrenocortical activity. Increases, decreases, and no effect have been reported (see Brain \& Benton, 1979, for a review of this area).

Group-housed animals are also presumably stressed because of agonistic encounters, yet they had lower latencies than did individually housed animals. If dominance influences the degree of stress from these encounters and submissive animals experience the greatest stress, one might expect greater variance in latency within the grouped animals based on greater individual differences in stress, but this was not the case. It may be that by the time at which the subjects were tested (after 15 weeks or 1 month in a housing condition), the frequency of agonistic encounters had declined as the subjects developed stable relations (Bernstein, 1981). This question might be addressed by examining encounters and nociceptive latencies at various points after differential housing.

\section{REFERENCES}

Basbaum, A. I., \& Fields, H. L. (1984). Endogenous pain control systems: Brainstem spinal pathways and endorphin circuitry. Annual Review of Neuroscience, 7, 309-338.

BernsteIn, I. S. (1981). Dominance: The baby and the bathwater. Behavioral \& Brain Sciences, 4, 419-429.

BraIN, P. F., \& BENTON, D. (1979). The interpretation of physiological correlates of differential housing in laboratory rats. Life Sciences, 24, 99-116.

Brain, P. F., \& Benton, D. (1983). Conditions of housing, hormones, and aggressive behavior. In B. B. Svare (Ed.), Hormones and aggressive behavior (pp. 351-372). New York: Plenum.

Dalrymple-Alford, J. C., \& Benton, D. (1984). Behavioral inhibition and the age at social isolation in rats. Quarterly Journal of Experimental Psychology, 36B, 27-38.

EINON, D. F. (1980). Spatial memory and response strategies in rats: Age, sex and rearing differences in performance. Quarterly Journal of Experimental Psychology, 32, 473-489.

EINON, D. F., \& SAHAKIAN, B. J. (1979). Environmentally induced differences in susceptibility of rats to CNS stimulants and CNS depressants: Evidence against a unitary explanation. Psychopharmacology, 61, 299-307.

Gentsch, C., Lichtsteiner, M., Frischinecht, H.-R., Feer, H., \& Siegfried, B. (1988). Isolation-induced locomotor hyperactivity and hypoalgesia in rats are prevented by handling and reversed by resocialization. Physiology \& Behavior, 43, 13-16.
Goldsmith, J. F., Brain, P. F., \& Benton, D. (1978). Effects of age at differential housing and the duration of individual housing/grouping on intermale fighting behavior and adrenocortical activity in TO strain mice. Aggressive Behavior, 2, 307-323.

Gordon, C. J. (1990). Thermal biology of the laboratory rat. Physiology \& Behavior, 47, 963-991.

GraU, J. W. (1987). Activation of the opioid and nonopioid analgesic systems: Evidence for a memory hypothesis and against the coulometric hypothesis. Journal of Experimental Psychology: Animal Behavior Processes, 13, 215-225.

JACKSON, R. L., MAIER, S. F., \& CoON, D. J. (1979). Long-term analgesic effects of inescapable shock and learned helplessness. Science, 206, 91-93.

Lessac, M. S., \& Solomon, R. L. (1969). Effects of early isolation on the later behavior of beagles: A methodological demonstration. Developmental Psychology, 1, 14-25.

MelzacK, R., \& SCOTT, T. H. (1957). The effects of early experience on the response to pain. Journal of Comparative \& Physiological Psychology, 50, 156-161.

Morgan, M. J., Einon, D. F., \& Nicholas, D. (1975). The effects of isolation rearing on behavioral inhibition in the rat. Quarterly Journal of Experimental Psychology, 27, 615-634.

Ness, T. J., \& Gebhart, G. F. (1986). Centrifugal modulation of the rat tail reflex evoked by graded noxious heating of the tail. Brain $R e-$ search, 386, 41-52.

NishikaWA, T., \& TANAKA, M. (1978). Altered behavioral responses to intense foot shock in socially-isolated rats. Pharmacology, Biochemistry \& Behavior, 8, 61-67.

Pugliosi-Allegra, S., \& Oliverio, A. (1983). Social isolation: Effects on pain threshold and stress-induced analgesia. Pharmacology, Biochemistry \& Behavior, 19, 679-681.

WADE, S. E., \& MAIER, S. F. (1986). Effects of individual housing and stressor exposure upon the acquisition of watermaze escape. Learning \& Motivation, 17, 287-310.

WATKINS, L. R., \& MAYER, D. J. (1982). Organization of endogenous opiate and nonopiate pain control systems. Science, 216, 1185-1192.

\section{NOTES}

1. In an attempt to examine differences in pain sensitivity more closely, a post hoc measurement of dominance was conducted with the grouped subjects, using a dyadic food competition task. Dominance rank accounted for virtually none of the variance.

2. The Mauchley test for sphericity of the variance-covariance matrices was rejected for the Phase 2 data of the housing experiment $\left[\chi^{2}(14)=32.21, p<.01\right]$. To compensate for this violation of the assumptions of a repeated measures design, the degrees of freedom for within-subject variance components (trials and all of the interactions with trials) were multiplied by the Greenhouse-Geisser correction, 0.686 The analysis with these reduced degrees of freedom produced a pattern of results identical to that obtained with the simple $F$ ratios reported in the text. 